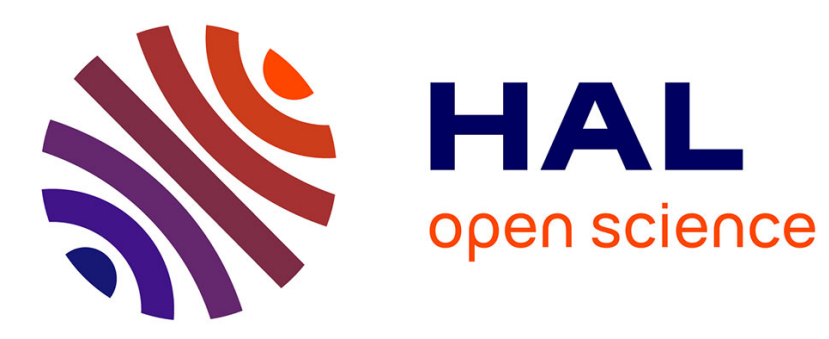

\title{
Localized time-dependent perturbations in metals : formalism and simple examples
}

\author{
Annie Blandin, A. Nourtier, D.W. Hone
}

\section{To cite this version:}

Annie Blandin, A. Nourtier, D.W. Hone. Localized time-dependent perturbations in metals: formalism and simple examples. Journal de Physique, 1976, 37 (4), pp.369-378. 10.1051/jphys:01976003704036900 . jpa-00208432

\section{HAL Id: jpa-00208432 https://hal.science/jpa-00208432}

Submitted on 1 Jan 1976

HAL is a multi-disciplinary open access archive for the deposit and dissemination of scientific research documents, whether they are published or not. The documents may come from teaching and research institutions in France or abroad, or from public or private research centers.
L'archive ouverte pluridisciplinaire HAL, est destinée au dépôt et à la diffusion de documents scientifiques de niveau recherche, publiés ou non, émanant des établissements d'enseignement et de recherche français ou étrangers, des laboratoires publics ou privés. 


\title{
LOCALIZED TIME-DEPENDENT PERTURBATIONS IN METALS : FORMALISM AND SIMPLE EXAMPLES
}

\author{
A. BLANDIN, A. NOURTIER \\ Laboratoire de Physique des Solides, Université Paris-Sud, Centre d’Orsay, 91405 Orsay, France \\ and D. W. HONE \\ Physics Department, University of California, Santa Barbara, California 93106, U.S.A.
}

(Reçu le 10 octobre 1975, accepté le 17 décembre 1975)

\begin{abstract}
Résumé. - La méthode introduite par Keldysh pour traiter les problèmes hors d'équilibre est appliquée au cas de fortes perturbationes, localisées, dépendant du temps, dans les métaux. Après avoir introduit le formalisme, nous traitons quelques exemples simples qui sont liés à la dynamique des atomes près des surfaces : probabilités d'ionisation d'atomes quittant une surface métallique, coefficients de frottement d'atomes au voisinage d'une surface métallique.
\end{abstract}

\begin{abstract}
Methods introduced by Keldysh to treat non-equilibrium problems are applied to strong, localized time-dependent perturbations in metals. After having introduced the formalism, we treat simple examples which are linked to the dynamics of atoms near surfaces : ionization probabilities of atoms leaving a metallic surface and friction coefficients of atoms near a metallic surface.
\end{abstract}

1. Introduction. - Numerous interesting problems can be viewed as involving strong time-dependent perturbations in an essential way. A first example is given by moving particles (such as protons or ions) inside a metal or near the surface of a metal. The total Hamiltonian of the system can be written :

$$
\mathscr{H}=\mathscr{H}_{0}+\mathfrak{H}_{1}[\mathbf{R}(t)]
$$

where $\mathfrak{H}_{1}$ is the perturbation created by the moving particle, the position of which is $\mathbf{R}(t)$ at time $t$. If one knows $\mathbf{R}(t)$, the perturbation $\mathscr{H}_{1}$ becomes a function of time $t$. Typical experiments of this kind are :

1) Charge exchange of particles reflected by metallic surfaces or crossing thin foils.

2) Ionization probabilities of atoms extracted from metals by ionic bombardment.

In these cases, the interaction $\mathscr{H}_{1}$ is a strong timedependent perturbation which cannot be treated by linear response theory. A very approximate treatment of problems of this kind was given many years ago [1]. Here we present a rigorous method which is based on a theory of non-equilibrium processes, namely the Keldysh formalism [2]. This formalism is the starting point of all the theoretical development of this paper.

Another example of a time-dependent perturbation is the problem of friction of atoms near metallic surfaces ; this includes the case of desorption, adsorption or reflection of atoms as well as the case of diffusion or catalysis on metals. This problem can be discussed directly using the fluctuation-dissipation theory which relates the friction force to the correlation function of the generalized force, $-\partial \cdot \mathcal{K} / \partial R$, of the problem. Here we present an alternative calculation of the friction force based on the Keldysh method.

In all these problems one could follow, in principle, a two-step approach : first one treats the effect of the time-dependent perturbation $]_{1}$ on the conduction electrons and deduces the energy $E(t)$ of the system, the derivative of which is just the force $F$ on the particle multiplied by $(-u)$, where $u$ is the velocity of the particle. Second, one can feed the force $F$ back into the equation of motion of the particle, which, in principle, determines $R(t)$ by a self-consistent method. In the study of friction forces, this amounts to the introduction of the friction force into a FokkerPlanck equation and to the solution of this equation. In this paper, we shall concentrate on the first part of this program - that is, the study of the response of the electrons to the time-dependent perturbation.

A last example of strong time-dependent perturbations is given by the X-ray absorption (or emission) in metals. In that case, the perturbing center is fixed; the problem can be described by the sudded creation (or annihilation) of a hole in one of the deep levels of 
one atom in the metal. The presence of this deep hole gives rise to screening by the conduction electrons. These electrons thus experience localized scattering when the hole is present and no scattering when there is no hole. We are faced with the problem of a localized time-dependent perturbation which is suddenly switched on (because the absorption - or emission - process, is very fast). The $\mathrm{X}$-ray problem has been widely discussed [3] : the main result of these studies is the existence of threshold singularities in the absorption (or emission) spectra. In spite of the obvious similarities here to the physical situations with which we are concerned in this work, the study of these singularities is not usefully made by the methods we will discuss. However, later we will return to the problem briefly to clarify the relationship of our work to it.

In section 2 we give a brief review of the Keldysh method. Although nothing new is introduced in this section, we include it because the method is not widely known. We apply the results in section 3 to the case of a time-dependent scattering potential and we discuss the relation between the results obtained and the X-ray problem. In section 4, we apply the method to a simple Hamiltonian which describes the ionization probability of an atom leaving a metallic surface. The last section is devoted to a discussion of friction coefficients.

Preliminary results have been published in a recent letter [4]. In this paper, we shall emphasize the methods rather than the detailed results and the comparison with experiments. We shall also restrict ourselves to the case of non-interacting electrons. Detailed papers applying the results to particular physical situations will be published in the near future : one will deal with ionization probabilities of atoms leaving a surface; the second will be devoted to the calculation of friction coefficients for adsorption or for simple reactions on surfaces.

2. The Keldysh method for non-equilibrium processes. - Consider a system described by the Hamiltonian :

$$
\mathscr{H}=\mathscr{H}_{0}+\mathscr{H}_{1}(t) .
$$

The linear response of the system to $\mathscr{H}_{1}(t)$ may be found by ordinary perturbation theory, in terms of correlation functions for the system in thermodynamic equilibrium under.$K_{0}$. But, if the perturbation is sufficiently strong or its time dependence such that the system is far from equilibrium, then new techniques must be used. Such is the case for the transient (irreversible) response to a time-dependent potential, the problem which we treat in this paper.

2.1 GeNERAL FORMULATION. - The method consists of an extension of the usual diagrammatic techniques for calculating Green's functions. For two-time Green's functions (we consider here only zero temperature) :

$$
G\left(t, t^{\prime}\right)=-i\left\langle\psi(0)\left|T\left[A(t) B^{+}\left(t^{\prime}\right)\right]\right| \psi(0)\right\rangle
$$

where the Fermion operators $A(t)$ and $B^{+}(t)$ are in the Heisenberg representation and $T$ is the usual chronological ordering operator. It is clear that one cannot simply modify ordinary diagrammatic perturbation theory by attaching the time dependence of $\pi_{1}(\tau)$ to each vertex at time $\tau$. The ordinary diagrams include vertices at all times later than $t$ and $t^{\prime}$ whereas the manifestly causal function (3) must clearly be independent of the behaviour of $\pi_{1}(\tau)$ for $\tau>t, t^{\prime}$.

This difficulty is clearly seen if we analyze the usual perturbation theory. In the interaction representation, the state $|\psi(t)\rangle$ evolves according to :

$$
|\psi(t)\rangle=U\left(t, t^{\prime}\right)\left|\psi\left(t^{\prime}\right)\right\rangle
$$

where the evolution operator $U\left(t, t^{\prime}\right)$ is defined by :

$$
U\left(t, t^{\prime}\right)=T \exp \left[-i \int_{t^{\prime}}^{t} \tilde{\mathscr{K}}_{1}(\tau) \mathrm{d} \tau\right] .
$$

The time dependence of the operator $\tilde{A}$ in this representation is governed only by $\mathrm{X}_{0}$ :

$$
\tilde{A}=\mathrm{e}^{i \pi e_{\mathrm{o}} t} A \mathrm{e}^{-i 3 e_{\mathrm{o}} t} \text {. }
$$

Then, $G\left(t, t^{\prime}\right)$ is given as :

$$
G\left(t, t^{\prime}\right)=-i\left\langle 0\left|S^{+} T\left[\tilde{A}(t) \widetilde{B}^{+}\left(t^{\prime}\right) S\right]\right| 0\right\rangle
$$

where the $S$-matrix $S \equiv U(+\infty,-\infty)$ and

$$
|0\rangle \equiv|\tilde{\psi}(-\infty)\rangle \text {. }
$$

In the absence of irreversible effects (in particular $\mathcal{H}_{1}(t)$ switches on at $t \rightarrow-\infty$ and off at $t \rightarrow+\infty$ : $\left.\mathcal{X}_{1}(t)=\mathcal{K}_{1} \mathrm{e}^{-\eta|t|}, \eta \rightarrow 0^{+}\right)$, the adiabatic theorem implies :

$$
<0\left|S^{+}=\mathrm{e}^{i \alpha}<0\right|
$$

where $\alpha$ is a real number. Then :

$$
G\left(t, t^{\prime}\right)=-i \frac{\left\langle 0\left|T\left[\tilde{A}(t) \tilde{B}^{+}\left(t^{\prime}\right) S\right]\right| 0\right\rangle}{\langle 0|S| 0\rangle}
$$

which, with Wick's theorem, leads to the ordinary diagrammatic perturbation expansion and the linked cluster theorem.

It is the final step (7) which cannot be taken in the presence of irreversible effects, because the system is then not returned to the ground state at $t \rightarrow+\infty$. However, $G\left(t, t^{\prime}\right)$ is still given correctly by eq. (6) as a ground state average.

The generalization amounts to writing (6) as the average of a suitably defined ordered product of 
operators, so that diagrammatic techniques can be used.

Let us define a contour (Fig. 1) from $-\infty$ to $\mathscr{C}$ and thence from $\mathfrak{C}$ to $-\infty$. The time $\mathfrak{C}$ is greater than $t$ and $t^{\prime}$ and can be taken as $+\infty$. Let $s$ be the curvilinear

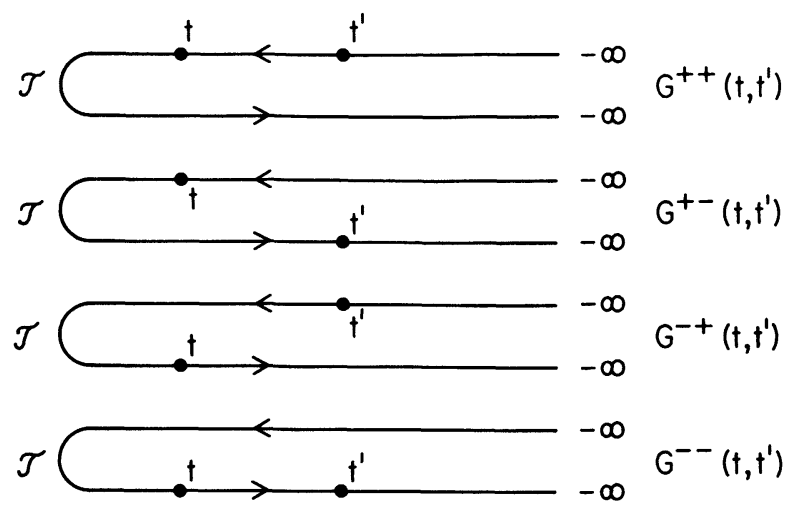

FIG. 1. - The four functions $G^{\alpha \beta}\left(t, t^{\prime}\right)$, as defined with $t$ and $t^{\prime}$ on the increasing or decreasing branches of the contour $\mathrm{C}$.

abscissa along the contour $\mathrm{C}$. We have $s=t$ on the increasing branch of the contour and $s=2 \mathfrak{C}-t$ on the decreasing branch. One can define :

$$
\mathbf{G}\left(s, s^{\prime}\right)=-i\left\langle 0\left|T_{\mathrm{c}}\left[\tilde{A}(s) \tilde{B}^{\dagger}\left(s^{\prime}\right) S_{\mathrm{c}}\right]\right| 0\right\rangle
$$

$T_{\mathrm{c}}$ orders the abscissas on the contour and one has for $S_{\mathrm{c}}$ :

$$
S_{\mathrm{c}}=U(-\infty, \mathfrak{G}) U(\mathfrak{G},-\infty)
$$

In the ordering of $S_{\mathrm{c}}$, one must keep all the terms coming from $U(-\infty, \mathfrak{C})$ on the decreasing branch of the contour; these terms appear to the left of the terms coming from $U(\mathcal{C},-\infty)$ which are on the increasing branch.

Specifying the branches involved for $s$ and $s^{\prime}$ in eq. (9) gives rise to four functions $G^{\alpha \beta}\left(t, t^{\prime}\right)$ where $\alpha$ and $\beta$ are + or - depending on the positions of $s$ and $s^{\prime}$ on the increasing $(+)$ or decreasing $(-)$ part of the contour $\left({ }^{1}\right)$ (see Fig. 1). The relation between $s$ (or $s^{\prime}$ ) and $t$ (or $t^{\prime}$ ) has been given above. One has :

$$
\begin{aligned}
& G^{\alpha \beta}\left(t, t^{\prime}\right)= \\
& \left\{\begin{array}{c}
-i\langle 0| U(-\infty, t) \tilde{A}(t) U\left(t, t^{\prime}\right) \tilde{B}^{+}\left(t^{\prime}\right) \times \\
\times U\left(t^{\prime},-\infty\right)|0\rangle, \text { if } s>s^{\prime} \\
i\langle 0| U\left(-\infty, t^{\prime}\right) \tilde{B}^{+}\left(t^{\prime}\right) U\left(t^{\prime}, t\right) \tilde{A}(t) \times \\
\times U(t,-\infty)|0\rangle, \text { if } s<s^{\prime} .
\end{array}\right.
\end{aligned}
$$

$G^{++}\left(t, t^{\prime}\right)$ is identical to $G\left(t, t^{\prime}\right)$ as defined by eq. (3) or (6). It is also clear that there are only two independent functions. One has :

(') In this paper we will not use Keldysh's unconventional notation, which may be misleading. Also, we omit, in the present section, indices $\mathbf{k}, \mathbf{k}^{\prime}, \ldots$, which label the operators $A$ and $B$ in the Green's function.

$$
\begin{gathered}
G^{++}\left(t, t^{\prime}\right)=\theta\left(t-t^{\prime}\right) G^{-+}\left(t, t^{\prime}\right)+ \\
+\theta\left(t^{\prime}-t\right) G^{+-}\left(t, t^{\prime}\right) \\
G^{--}\left(t, t^{\prime}\right)=\theta\left(t-t^{\prime}\right) G^{+-}\left(t, t^{\prime}\right)+ \\
+\theta\left(t^{\prime}-t\right) G^{-+}\left(t, t^{\prime}\right) .
\end{gathered}
$$

Thus only $G^{-+}$and $G^{+-}$are independent. One has also :

$$
G^{++}+G^{--}=G^{+-}+G^{-+} .
$$

Wick's theorem and diagrammatic techniques can now be applied to the Green's function (9). There exists a linked cluster theorem and one can introduce a self-energy and a Dyson equation.

The self-energy $\Sigma\left(s, s^{\prime}\right)$ is defined on the contour $\mathrm{C}$. Like $G$ itself $\Sigma$ is constructed from four functions $\Sigma^{\alpha \beta}\left(t, t^{\prime}\right)$ depending on the positions of $s$ and $s^{\prime}$ on the contour. Furthermore, one can show :

$$
\Sigma^{++}+\Sigma^{--}=-\left(\Sigma^{+-}+\Sigma^{-+}\right) \text {. }
$$

The Dyson equation on the contour is :

$$
\begin{aligned}
& \mathbf{G}\left(s, s^{\prime}\right)=\mathbf{G}_{0}\left(s, s^{\prime}\right)+\iint \mathbf{G}_{0}\left(s, s^{\prime \prime}\right) \Sigma\left(s^{\prime \prime}, s^{\prime \prime \prime}\right) \\
& \mathbf{G}\left(s^{\prime \prime \prime}, s^{\prime}\right) \mathrm{d} s^{\prime \prime} \mathrm{d} s^{\prime \prime \prime}
\end{aligned}
$$

where $G_{0}$ is the unperturbed Green's function defined on the contour $C$. One can use $2 \times 2$ matrices to describe $G^{\alpha \beta}\left(t, t^{\prime}\right)$ and $\Sigma^{\alpha \beta}\left(t, t^{\prime}\right)$. The Dyson equation becomes

$$
\overline{\bar{G}}=\overline{\bar{G}}_{0}+\overline{\bar{G}}_{0} \overline{\bar{\Sigma}} \overline{\bar{G}}
$$

where integrations over intermediate times are implicit. For simplicity we shall use this notation in the following sections.

Explicit reduction of eq. (14) to independent equations is effected by the canonical transformation :

$$
\overline{\bar{A}}=\frac{1}{2}\left(\begin{array}{rr}
1 & -1 \\
1 & 1
\end{array}\right) \overline{\bar{A}}\left(\begin{array}{rr}
1 & 1 \\
-1 & 1
\end{array}\right) .
$$

Thus, the matrix Green's function becomes :

$$
\overline{\overline{\mathfrak{G}}}=\left(\begin{array}{cc}
0 & G^{\mathrm{r}} \\
G^{\mathrm{a}} & F
\end{array}\right)
$$

where the retarded and advanced functions are $\left({ }^{2}\right)$ :

$$
\left.\begin{array}{l}
G^{\mathbf{r}}=G^{++}-G^{+-} \\
G^{\mathrm{a}}=G^{++}-G^{-+}
\end{array}\right\}
$$

$G^{\mathrm{r}}$ and $G^{\mathrm{a}}$ contain equivalent information; one is essentially the Hermitian conjugate of the other. The function $F$ is given by :

$$
F=G^{++}+G^{--} \text {. }
$$

(2) Here we use the standard notation for retarded and advanced functions, which is the opposite of the convention chosen by Keldysh [2]. 
The self-energy becomes the matrix :

$$
\overline{\overline{\mathcal{V}}}=\left(\begin{array}{cc}
\Omega & \Sigma^{\mathrm{a}} \\
\Sigma^{\mathrm{r}} & 0
\end{array}\right)
$$

where :

$$
\left.\begin{array}{l}
\Sigma^{\mathbf{r}}=\Sigma^{++}+\Sigma^{+-} \\
\Sigma^{\mathbf{a}}=\Sigma^{++}+\Sigma^{-+} \\
\Omega=\Sigma^{++}+\Sigma^{--}
\end{array}\right\}
$$

The components of the Dyson equation can now be written as :

$$
\begin{gathered}
G^{\mathrm{r}}=G_{0}^{\mathrm{r}}+G_{0}^{\mathrm{r}} \Sigma^{\mathrm{r}} G^{\mathrm{r}} \\
G^{\mathrm{a}}=G_{0}^{\mathrm{a}}+G_{0}^{\mathrm{a}} \Sigma^{\mathrm{a}} G^{\mathrm{a}} \\
F=F_{0}+G_{0}^{\mathrm{a}} \Omega G^{\mathrm{r}}+G_{0}^{\mathrm{a}} \Sigma^{\mathrm{a}} F+F_{0} \Sigma^{\mathrm{r}} G^{\mathrm{r}}
\end{gathered}
$$

2.2 ONE BODY TIME-DEPENDENT PERTURBATION $\left({ }^{3}\right)$. - If we restrict the discussion to the case ${ }^{-}$of a onebody potential $V(t)$ we can simplify the preceding discussion. The self-energy matrix becomes :

$$
\overline{\bar{V}}\left(t, t^{\prime}\right)=\delta\left(t-t^{\prime}\right)\left(\begin{array}{cc}
V(t) & 0 \\
0 & -V(t)
\end{array}\right) .
$$

With this self-energy matrix, one can easily see, using the Dyson eq. (14) that the Green's function $G\left(t, t^{\prime}\right)$ does not depend on $V(\tau)$ for times $\tau$ larger than $t$ and $t^{\prime}$. Due to the minus sign in eq. (22), there is an exact cancellation of the contributions between $\operatorname{Sup}\left(t, t^{\prime}\right)$ and $\mathcal{C}$. Thus, the value of $\mathcal{C}$ does not matter, as long as it is larger than $t$ and $t^{\prime}$. We verify, in this simple case, that the theory satisfies the requirements of causality.

The canonical transformation gives for the selfenergy matrix :

$$
\overline{\bar{v}}\left(t, t^{\prime}\right)=\delta\left(t-t^{\prime}\right)\left(\begin{array}{cc}
0 & V(t) \\
V(t) & 0
\end{array}\right)
$$

and the Dyson eq. (21) become :

$$
\left.\begin{array}{l}
G^{\mathrm{r}}=G_{0}^{\mathrm{r}}+G_{0}^{\mathrm{r}} V G^{\mathrm{r}} \\
G^{\mathrm{a}}=G_{0}^{\mathrm{a}}+G_{0}^{\mathrm{a}} V G^{\mathrm{a}} \\
F=F_{0}+F_{0} V G^{\mathrm{r}}+G_{0}^{\mathrm{a}} V F
\end{array}\right\} .
$$

For such a one-body potential (in the many electron system of the Fermi sea of a metal) $G^{\mathrm{a}}$ and $G^{\mathrm{r}}$ satisfy

$\left({ }^{3}\right)$ For a one-body perturbation, the function $|\psi(t)\rangle$ which describes the system can be written as a Slater determinant made up with one-electron functions $\varphi_{i}$ satisfying :

$$
\left[\mathscr{H}_{0}+\mathscr{H}_{1}(t)\right] \varphi_{i}(\mathbf{r}, t)=i \frac{\partial \varphi_{i}}{\partial t}(\mathbf{r}, t)
$$

with appropriate initial conditions.

In principle, one can obtain all information from $|\psi(t)\rangle$.

In practice, the calculations quickly become intractable and use of the Keldysh method is much more rewarding. simple one particle equations. These equations do not involve the occupancy of the one-electron states and thus the existence of a Fermi level. $G^{\mathbf{a}}$ and $G^{\mathbf{r}}$ are then readily obtained. The many-body aspects of the problem reside in the function $F$. This point can be seen directly in the explicit formula for $F_{0}$ as given in the next section.

Furthermore, one can obtain a solution for $F$ in terms of $G^{\mathrm{r}}$ and $G^{\mathrm{a}}$ by an iterative solution of the last integral eq. (24). The result is :

$$
F=\left(1+G^{\mathrm{a}} V\right) F_{0}\left(1+V G^{\mathrm{r}}\right) .
$$

Eq. (24) and (25) form the basis for the discussion given in this paper.

3. Detailed discussion of a one body time-dependent potential. - Let us look in more detail at the case of a one-body potential within the Fermi sea of conduction electrons. The Hamiltonian is :

where :

$$
\mathscr{H}=\mathscr{H}_{0}+\mathscr{H}_{1}(t)
$$

$$
\left.\begin{array}{l}
\mathscr{H}_{0}=\sum_{k} \varepsilon_{k} c_{k}^{+} c_{k} \\
\mathscr{H}_{1}(t)=\sum_{k k^{\prime}} V_{k k^{\prime}}(t) c_{k}^{+} c_{k^{\prime}}
\end{array}\right\} .
$$

We have omitted explicit spin indices for simplicity. The results below are essentially unchanged if the indices $k, k^{\prime}$ are taken to represent both orbital and spin quantum numbers.

3.1 Calculation of THE Green's function. Instead of using the Green's functions $G_{k k^{\prime}}^{\alpha \beta}\left(t, t^{\prime}\right)$ (or $G^{\mathbf{a}}, G^{\mathbf{r}}, F$ ), we shall introduce the reduced Green's functions $g$ defined by :

$$
\begin{aligned}
& G_{k k^{\prime}}^{\alpha \beta}\left(t, t^{\prime}\right)= \\
&=\exp \left[i \int_{0}^{t} \varepsilon_{k} \mathrm{~d} \tau-i \int_{0}^{t^{\prime}} \varepsilon_{k^{\prime}} \mathrm{d} \tau\right] g_{k k^{\prime}}^{\alpha \beta}\left(t, t^{\prime}\right)
\end{aligned}
$$

and similar expressions for $G^{\mathrm{a}}, G^{\mathrm{r}}$ and $F$. We introduce also reduced unperturbed functions. Furthermore, we define :

$$
v_{k k^{\prime}}(t)=V_{k k^{\prime}}(t) \exp \left[i \int_{0}^{t}\left(\varepsilon_{k}-\varepsilon_{k^{\prime}}\right) \mathrm{d} \tau\right] .
$$

The unperturbed reduced Green's function matrix is :

$\overline{\bar{g}}_{0}=-i \delta_{k k^{\prime}}\left(\begin{array}{cc}\theta\left(t-t^{\prime}\right)-n_{k}^{0} & -n_{k}^{0} \\ 1-n_{k}^{0} & \theta\left(t^{\prime}-t\right)-n_{k}^{0}\end{array}\right)$

where $n_{k}^{0}$ are the Fermi occupation numbers and $\theta(t)$ is the Heaviside function.

The Dyson equation becomes, in matrix notation :

$$
\overline{\bar{g}}=\overline{\bar{g}}_{0}+\overline{\bar{g}}_{0} \bar{v} \bar{g} \text {. }
$$


We proceed as in the previous section with the introduction of the same unitary transformation. The transformed unperturbed Green's function matrix is :

$\left(\begin{array}{cc}0 & g_{0}^{\mathrm{r}} \\ g_{0}^{\mathrm{a}} & f_{0}\end{array}\right)=-i \delta_{k k^{\prime}}\left(\begin{array}{cc}0 & -\theta\left(t^{\prime}-t\right) \\ \theta\left(t-t^{\prime}\right) & 1-2 n_{k}^{0}\end{array}\right)$.

Eq. (31) clearly exhibits the fact that only the $f$ (here $f_{0}$ ) functions depend on the occupation numbers $n_{k}^{0}$.

The Dyson equations are, similarly to (24) and (25) :

$$
\left.\begin{array}{l}
g^{\mathrm{r}}=g_{0}^{\mathrm{r}}+g_{0}^{\mathrm{r}} v g^{\mathrm{r}} \\
g^{\mathrm{a}}=g_{0}^{\mathrm{a}}+g_{0}^{\mathrm{a}} v g^{\mathrm{a}} \\
f=\left(1+g^{\mathrm{a}} v\right) f_{0}\left(1+v g^{\mathrm{r}}\right)
\end{array}\right\}
$$

The last equation can be transformed, using the explicit expressions (31) for $f_{0}, g_{0}^{\mathrm{a}}$ and $g_{0}^{\mathrm{r}}$ :

$$
\begin{aligned}
f_{k k^{\prime}}\left(t, t^{\prime}\right)=i \sum_{q}(2 & \left.n_{q}^{0}-1\right) \times \\
& \times\left[\left(1+g^{\mathrm{a}} v\right) g_{0}^{\mathrm{a}}\right]_{k q}(t,-\infty) \\
& \times\left[g_{0}^{\mathrm{r}}\left(1+v g^{\mathrm{r}}\right)\right]_{q k^{\prime}}\left(-\infty, t^{\prime}\right)
\end{aligned}
$$

where the notation $[. .].\left(t, t^{\prime}\right)$ means that the first and last times involved in the bracket are $t$ and $t^{\prime}$. It follows from the equations obeyed by $g^{\mathrm{a}}$ and $g^{\mathrm{r}}$ that

$$
\begin{aligned}
f_{k k^{\prime}}\left(t, t^{\prime}\right)=i \sum_{q}\left(2 n_{q}^{0}-1\right) g_{k q}^{\mathrm{a}}( & t, \infty) \times \\
& \times g_{q k^{\prime}}^{\mathrm{r}}\left(-\infty, t^{\prime}\right) .
\end{aligned}
$$

Now, we obtain $g^{\alpha \beta}$ in terms of $g^{\mathrm{a}}$ and $g^{\mathrm{r}}$; in particular

$g_{k k^{\prime}}^{++}\left(t, t^{\prime}\right)=g_{k k^{\prime}}\left(t, t^{\prime}\right)$

$$
\begin{aligned}
& \quad=\frac{1}{2}\left[g_{k k^{\prime}}^{\mathrm{a}}\left(t, t^{\prime}\right)+g_{k k^{\prime}}^{\mathrm{r}}\left(t, t^{\prime}\right)+\right. \\
& \left.+i \sum_{q}\left(2 n_{q}^{0}-1\right) g_{k q}^{\mathrm{a}}(t,-\infty) g_{q k^{\prime}}^{\mathrm{r}}\left(-\infty, t^{\prime}\right)\right] .
\end{aligned}
$$

This expression can be simplified by using the fact $g^{\mathbf{a}}$ and $g^{\mathrm{r}}$ do not depend on $n_{q}^{0}$. Suppose first that $n_{q}^{0}=0$ for all $q$. Then from the definition (6) $g_{k k^{\prime}}\left(t^{\prime}, t\right)=0$ for $t^{\prime}>t$ and the above equation gives :

$$
g_{k k^{\prime}}^{\mathrm{r}}\left(t, t^{\prime}\right)=i \theta\left(t^{\prime}-t\right) \sum_{q} g_{k q}^{\mathrm{a}}(t,-\infty) g_{q k^{\prime}}^{\mathrm{r}}\left(-\infty, t^{\prime}\right)
$$

Similarly, by making $n_{q}^{0}=1$, one obtains the conjugate relation :

$$
\begin{aligned}
g_{k k^{\prime}}^{\mathrm{a}}\left(t, t^{\prime}\right)=-i \theta\left(t-t^{\prime}\right) \sum_{q} g_{k q}^{\mathrm{a}}(t,-\infty) & \times \\
& \times g_{q k^{\prime}}^{\mathrm{r}}\left(-\infty, t^{\prime}\right) .
\end{aligned}
$$

Using these two identities, $g$ can be written, $\varepsilon_{\mathrm{f}}$ being the Fermi energy, as

$$
g_{k k^{\prime}}\left(t, t^{\prime}\right)= \begin{cases}-i \sum_{\varepsilon_{q}>\varepsilon_{\mathrm{f}}} g_{k q}^{\mathrm{a}}(t,-\infty) g_{q k^{\prime}}^{\mathrm{r}}\left(-\infty, t^{\prime}\right) & t>t^{\prime} \\ i \sum_{\varepsilon_{q}<\varepsilon_{\mathrm{f}}} g_{k q}^{\mathrm{a}}(t,-\infty) g_{q k^{\prime}}^{\mathrm{r}}\left(-\infty, t^{\prime}\right) & t<t^{\prime}\end{cases}
$$

The same relation holds for $G, G^{\mathrm{a}}$ and $G^{\mathrm{r}}$ :

$$
G_{k k^{\prime}}\left(t, t^{\prime}\right)= \begin{cases}-i \sum_{\varepsilon_{q}>\varepsilon_{\mathrm{f}}} G_{k q}^{\mathrm{a}}(t,-\infty) G_{q k^{\prime}}^{\mathrm{r}}\left(-\infty, t^{\prime}\right) & t>t^{\prime} \\ i \sum_{\varepsilon_{q}<\varepsilon_{\mathrm{f}}} G_{k q}^{\mathrm{a}}(t,-\infty) G_{q k^{\prime}}^{\mathrm{r}}\left(-\infty, t^{\prime}\right) & t<t^{\prime}\end{cases}
$$

In these equations « $-\infty$ » implies the limit $\tau \rightarrow-\infty$ for the whole product $G_{k q}^{\mathrm{a}}(t, \tau) G_{q k^{\prime}}^{\mathrm{r}}\left(\tau, t^{\prime}\right)$.

Eq. (35) and (36) are the main results which we use throughout this paper : the calculation of the Green's function of interest $G_{k k^{\prime}}\left(t, t^{\prime}\right)$ is reduced to the calculation of the advanced and retarded functions which do not involve the existence of a Fermi level. The many-body features of $G_{k k^{\prime}}\left(t, t^{\prime}\right)$ appear only through the summations over $q$ as shown by eq. (36).

3.2 ORBITAL OCCUPANCY AND THE X-RAY ABSORPTION PROBLEM. - For a time-dependent perturbation $V(t)$ (which tends towards a constant value for $t \rightarrow+\infty$ ), it is well known that the overlap between the unperturbed ground state and the state at time $t$ varies as $t^{-\alpha}$ for large $t$, where $\alpha$ generally has a non-integer value related to the phase-shifts due to the final potential $V(+\infty)$. This behaviour is the basis of the threshold singularities in the X-ray spectra as shown by Nozières and de Dominicis [3]. Here, we want to show that, on the contrary, other physical quantities are quite well-behaved for large times.

Let us consider, for example, the Wannier orbital which is centered at the origin. Its creation operator is :

$$
c_{0}^{+}=\frac{1}{\sqrt{N}} \sum_{k} c_{k}^{+}
$$


We are interested in the occupation number $n_{0}(t)$ when a sudden time-dependent perturbation occurs at time $t=0$ :

$$
V_{k k^{\prime}}(t)=-\frac{V}{N} \theta(t)
$$

This perturbation can be viewed as the potential due to the creation of a deep hole by X-ray absorption. We have for $n_{0}(t)$ :

$$
\begin{aligned}
n_{0}(t) & =\left\langle c_{0}^{+}(t) c_{0}(t)\right\rangle=\frac{-i}{N} \sum_{k k^{\prime}} G_{k k^{\prime}}(t, t+) \\
& =\frac{1}{N} \sum_{\varepsilon_{q}<\varepsilon_{\mathrm{r}}}\left|\sum_{k} G_{k q}^{\mathrm{a}}(t,-\infty)\right|^{2} .
\end{aligned}
$$

For $t$ and $t^{\prime}<0$ :

$$
\begin{array}{r}
G_{k k^{\prime}}^{\mathrm{a}}\left(t, t^{\prime}\right)=G_{0 k k^{\prime}}^{\mathrm{a}}\left(t, t^{\prime}\right)= \\
=-i \delta_{k k^{\prime}} \theta\left(t-t^{\prime}\right) \mathrm{e}^{-i \varepsilon_{k k^{\prime}\left(t-t^{\prime}\right)}} .
\end{array}
$$

For $t$ and $t^{\prime}>0, G_{k k^{\prime}}^{\mathrm{a}}\left(t, t^{\prime}\right)$ is a function of $\left(t-t^{\prime}\right)$. It is the Green's function which corresponds to a constant potential $V$. Its Fourier transform is easily calculated. We will need the quantity :

where :

$$
\sum_{k, k^{\prime}} G_{k k^{\prime}}^{\mathbf{a}}(\omega)=\Gamma(\omega)=\frac{\gamma(\omega)}{1+V \gamma(\omega)}
$$

$$
\gamma(\omega)=\sum_{k} \frac{1}{\omega-\varepsilon_{k}+i 0} .
$$

For $t>0$ and $t^{\prime}<0$ one obtains, using the Dyson equation,

$$
\begin{aligned}
G_{k k^{\prime}}^{\mathrm{a}}\left(t, t^{\prime}\right)= & -i \mathrm{e}^{i \varepsilon_{k^{\prime}}\left(t-t^{\prime}\right)} \times \\
& \times\left[\delta_{k k^{\prime}}-V \int_{0}^{t} \mathrm{~d} \tau \mathrm{e}^{i \varepsilon_{k^{\prime}} \tau} \sum_{q} G_{k q}^{\mathrm{a}}(\tau)\right] .
\end{aligned}
$$

This leads to the following expression for $n_{0}(t)$ :

$$
n_{0}(t)=\int^{e_{\mathrm{f}}} \rho(\varepsilon) \mathrm{d} \varepsilon\left|1-V \int_{0}^{t} \mathrm{~d} \tau \mathrm{e}^{i \varepsilon \tau} \Gamma(\tau)\right|^{2}
$$

where $\rho(\varepsilon)$ is the density of states of the band and $\Gamma(\tau)$ the Fourier transform of $\Gamma(\omega)$.

This result gives for $n_{0}(+\infty)$ the occupation number for a time independent potentiel $V$ as it should. Without giving a detailed expression for $n_{0}(t)$, one sees that $n_{0}(t)$ tends toward $n_{0}(+\infty)$ with a regular behaviour which does not involve terms of the type $t^{-\alpha}$, with $\alpha$ taking on either non-integer or integer values. In particular, contrary to previous suggestions [5] there is no $t^{-1}$ behaviour (as can be seen easily to first order in $V$ ).

Mathematically the difference can be explained as follows. When we look for $n_{0}(t)$, the potential $V$ appears on the contour as shown in figure $2 a$ in bold print. In the X-ray problem, when absorption occurs (a)

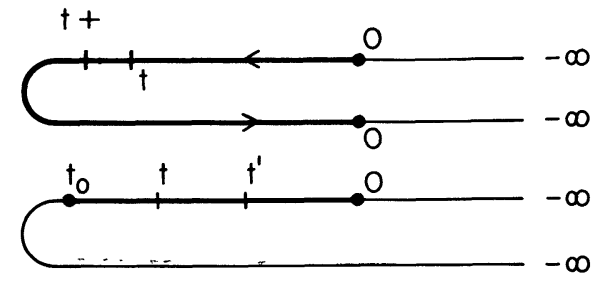

FIG. 2. - (a) The contour $\mathrm{C}$ as used for the calculation of $n_{\mathrm{d}}(t)$. (b) The contour $\mathrm{C}^{\prime}$ used in the X-ray problem.

at time $t=0$ the conduction electrons experience the same time-dependent perturbation (38).

The attractive potential $V$ is created by the deep hole. The Fourier transform of the absorption spectral density involves the calculation of a function $\varphi\left(t, t^{\prime}\right)$ defined on the contour $\mathrm{C}^{\prime}$ shown in bold print in figure $2 b$ [3]. It is not a causal function and exhibits singularities; to first order one obtains a logarithmic term ; summing up to all orders, one obtains a singular behaviour $t_{0}^{-\alpha}$ where $\alpha$ is a constant when $t$ and $t^{\prime}$ go to 0 and $t_{0}$, respectively. The constant $\alpha$ is related to the phase shift associated with scattering from the perturbation (38) as $t \rightarrow \infty$.

In conclusion, one can say that there is no contradiction between the results (44) and the X-ray problem : the difference arises due to the different nature of the quantities being calculated.

4. Ionization probability : an example. - Let us consider now the problem of ionization probabilities. A simple model Hamiltonian is provided by the Anderson Hamiltonian without interaction between electrons, but with time dependent parameters $\varepsilon_{\mathrm{d}}(t)$ and $V_{\mathrm{d} k}(t)$. We suppose that an atom leaving a surface makes $V_{\mathrm{d} k}(t)$ tend to zero as $t \rightarrow \infty$. Omitting spin indices as before, we have

$$
\begin{aligned}
\mathscr{H}=\sum_{k} \varepsilon_{k} n_{k}+\varepsilon_{\mathrm{d}}(t) & n_{\mathrm{d}}+ \\
& +\sum_{k}\left[V_{\mathrm{d} k}(t) c_{\mathrm{d}}^{+} c_{k}+\text { c.c. }\right]
\end{aligned}
$$

and we look for the d-level occupation number

$$
n_{\mathrm{d}}(t)=\left\langle c_{\mathrm{d}}^{+}(t) c_{\mathrm{d}}(t)\right\rangle=-i g_{\mathrm{dd}}(t, t+) .
$$

4.1 General Formulation. - With the previous notations, we write

$$
v_{k}(t)=V_{\mathrm{d} k}(t) \exp \left[i \int_{0}^{t} \varepsilon_{\mathrm{d}}(\tau) \mathrm{d} \tau-i \varepsilon_{k} t\right] .
$$

Decomposing the Dyson equation for $g^{\mathrm{a}}$, we get, in particular :

$$
\begin{aligned}
& g_{\mathrm{dd}}^{\mathrm{a}}=g_{0}^{\mathrm{a}}+\sum_{k} g_{0}^{\mathrm{a}} v_{k} g_{k \mathrm{~d}}^{\mathrm{a}} \\
& g_{k \mathrm{~d}}^{\mathrm{a}}=g_{0}^{\mathrm{a}} v_{k}^{*} g_{\mathrm{dd}}^{\mathrm{a}} .
\end{aligned}
$$


These two equations combine to give a Dyson equation for $g_{\mathrm{dd}}^{\mathrm{a}}$ :

where

$$
g_{\mathrm{dd}}^{\mathrm{a}}=g_{0}^{\mathrm{a}}+g_{0}^{\mathrm{a}} \sigma g_{\mathrm{dd}}^{\mathrm{a}}
$$

$$
\sigma=\sum_{k} v_{k} g_{0}^{\mathrm{a}} v_{k}^{*}
$$

We need $g_{\mathrm{dd}}$, which is given by (35) in terms of $g_{\mathrm{d} k}^{\mathrm{a}}$, $g_{k \mathrm{~d}}^{\mathrm{r}}$ and $g_{\mathrm{dd}}^{\mathrm{a} \text { r }}$. We can verify that

$$
g_{\mathrm{dd}}^{\mathrm{a}}(t,-\infty)=g_{\mathrm{dd}}^{\mathrm{r}}(-\infty, t)=0,
$$

as it should, since $n_{\mathrm{d}}(t)$ must not depend on the occupancy $n_{\mathrm{d}}^{0}$ of the d-level before it has been coupled to the electron gas. Using

$$
g_{\mathrm{d} k}^{\mathrm{a}}=g_{\mathrm{dd}}^{\mathrm{a}} v_{k} g_{0}^{\mathrm{a}},
$$

we obtain

$$
\begin{aligned}
n_{\mathrm{d}}(t)=\sum_{\varepsilon_{k}<\varepsilon_{\mathrm{f}}}\left|g_{\mathrm{d} k}^{\mathrm{a}}(t,-\infty)\right|^{2}= \\
\quad=\sum_{\varepsilon_{k}<\varepsilon_{\mathrm{f}}}\left|\int g_{\mathrm{dd}}^{\mathrm{a}}(t, \tau) v_{k}(\tau) \mathrm{d} \tau\right|^{2} .
\end{aligned}
$$

The problem is now to calculate $g_{\mathrm{dd}}^{\mathrm{a}}$, i.e., to solve the integral eq. (48). We shall make a first simplification, assuming that

$$
V_{\mathrm{d} k}(t)=V_{k} u(t) .
$$

Thus $\sigma$ can be written as

$$
\sigma\left(t, t^{\prime}\right)=u(t) u^{*}\left(t^{\prime}\right) \exp \left[i \int_{t^{\prime}}^{t} \varepsilon_{\mathrm{d}}(\tau) \mathrm{d} \tau\right] \Sigma\left(t-t^{\prime}\right)
$$

with

$$
\begin{aligned}
\Sigma(\tau)=-i \theta(\tau) \sum_{k}\left|V_{k}\right|^{2} \mathrm{e}^{-i \varepsilon_{k} \tau}= & \\
& =-i \theta(\tau) \int \mathrm{d} \varepsilon \frac{\Delta(\varepsilon)}{\pi} \mathrm{e}^{-i \varepsilon \tau}
\end{aligned}
$$

and

$$
\Delta(\varepsilon)=\pi \rho(\varepsilon)\left\langle\left|V_{k}\right|^{2}\right\rangle_{\varepsilon_{k}=\varepsilon},
$$

$\rho$ being the density of states of the electron gas. We now assume that $\Delta(\varepsilon)$ is independent of $\varepsilon$. Then :

where

$$
\Sigma(\tau)=-i \Delta \delta^{+}(\tau)
$$

$$
\delta^{+}(\tau)=2 \theta(\tau) \delta(\tau)
$$

which means a distribution localized in the vicinity of $\tau=0$ in the region $\tau>0$, the integral of which is unity. Then

$$
\sigma\left(t, t^{\prime}\right)=-i \delta^{+}\left(t-t^{\prime}\right) \Delta(t)
$$

where $\Delta(t)=\Delta|u(t)|^{2}$ appears as an instantaneous resonance width of the d-level.

The integral eq. (48) can now be solved; we obtain :

$$
g_{\mathrm{dd}}^{\mathrm{a}}\left(t, t^{\prime}\right)=-i \theta\left(t-t^{\prime}\right) \exp \left(-\int_{t^{\prime}}^{t} \Delta(\tau) \mathrm{d} \tau\right) .
$$

Putting this expression back into (50) gives the final result :

$$
\begin{aligned}
n_{\mathrm{d}}(t)= & \frac{\Delta}{\pi} \int_{-\infty}^{\varepsilon_{\mathrm{f}}} \mathrm{d} \varepsilon \mid \int_{-\infty}^{t} \mathrm{~d} \tau u(\tau) \times \\
& \times\left.\exp \left(i \int_{\tau}^{t}\left[\varepsilon-\varepsilon_{\mathrm{d}}\left(\tau^{\prime}\right)+i \Delta\left(\tau^{\prime}\right)\right] \mathrm{d} \tau^{\prime}\right)\right|^{2}
\end{aligned}
$$

Within some approximations, which are in fact those usually made, (58) gives an exact expression for the d-level occupancy. It exhibits important retardation effects, since it involves the instantaneous position and width of the d-level at all times before $t$.

4.9 Discussion. - For a slow variation of the parameters, the system will adiabatically follow the perturbation and the final result for $n_{\mathrm{d}}(\infty)$ will be 0 or 1 , depending on the sign of $\varepsilon_{\mathrm{f}}-\varepsilon_{\mathrm{d}}(\infty)$. If the variation is no longer slow, (58) must be used and $n_{\mathrm{d}}(\infty)$ may be different from 0 or 1 . This gives a simple example of ionization and the difference between $n_{\mathrm{d}}(\infty)$ and the adiabatic value $n_{\mathrm{d}}^{\text {ad }}$ provides an expression for the ionization probability.

Two cases arise :

1) The integral $\int^{\infty} \Delta\left(\tau^{\prime}\right) \mathrm{d} \tau^{\prime}$ diverges; i.e., $\Delta(t)$ decreases no faster than $1 / t$ at infinity. Then, when $t \rightarrow \infty$, the only values of $\tau$ contributing to the $\tau$-integral in (58) will be at infinity; due to the phase factor $\exp i \int\left(\varepsilon-\varepsilon_{J}\left(\tau^{\prime}\right)\right) \mathrm{d} \tau^{\prime}$, only values of $\varepsilon$ close to $\varepsilon_{\mathrm{d}}(\infty)$ will contribute to the $\varepsilon$-integral. Thus $n_{\mathrm{d}}(\infty)$ will not depend on the magnitude of $\varepsilon_{\mathrm{f}}-\varepsilon_{\mathrm{d}}(\infty)$ but only on its sign. More precisely, $n_{\mathrm{d}}(\infty)$ will be 1 if $\varepsilon_{\mathrm{f}}>\varepsilon_{\mathrm{d}}(\infty)$, and 0 if $\varepsilon_{\mathrm{f}}<\varepsilon_{\mathrm{d}}(\infty)$. This is the adiabatic result.

As an illustration of the behaviour of $n_{\mathrm{d}}(t)$; consider the limiting case $\Delta(\tau)=\alpha / \tau$ for $\tau>0$, with $\varepsilon_{\mathrm{d}}$ constant. Then

$$
n_{\mathrm{d}}(t)=\frac{\alpha}{\pi} \int_{-\infty}^{\left(\varepsilon_{\mathrm{f}}-\varepsilon_{\mathrm{d}}\right) t} \mathrm{~d} y\left|\int_{0}^{1} \mathrm{~d} x x^{\alpha-1 / 2} \mathrm{e}^{-i y x}\right|^{2}
$$

which depends only on $\alpha$ and $\left(\varepsilon_{\mathrm{f}}-\varepsilon_{\mathrm{d}}\right) t$. For instance, if $\alpha=\frac{1}{2}$,

$$
\begin{aligned}
& n_{\mathrm{d}}(t)=\frac{1}{2}-\frac{1}{\pi} \frac{1-\cos \left(\varepsilon_{\mathrm{f}}-\varepsilon_{\mathrm{d}}\right) t}{\left(\varepsilon_{\mathrm{f}}-\varepsilon_{\mathrm{d}}\right) t}+ \\
&+\frac{1}{\pi} \int_{0}^{\left(\varepsilon_{\mathrm{f}}-\varepsilon_{\mathrm{d}}\right) t} \mathrm{~d} x \frac{\sin x}{x} .
\end{aligned}
$$

When $t \rightarrow \infty, n_{\mathrm{d}}(t)$ tends towards its adiabatic value.

2) The integral $\int^{\infty} \Delta\left(\tau^{\prime}\right) \mathrm{d} \tau^{\prime}$ converges; i.e., $\Delta(t)$ decreases faster than $1 / t$. Then $n_{\mathrm{d}}(\infty)$ is obtained directly by putting $t=\infty$ in (58). In general, there 
is no reason for the result to be 0 or 1 , so that $n_{\mathrm{d}}(\infty)$ will depart from the adiabatic value.

As an example, suppose that $\Delta(t) \propto \mathrm{e}^{-\lambda t}$ with $\varepsilon_{\mathrm{d}}$ constant. Then

$n_{\mathrm{d}}(\infty)=\frac{1}{2}+\frac{1}{\pi} \int_{0}^{\infty} \frac{\mathrm{d} x}{x \cosh x} \sin \left(\frac{2\left(\varepsilon_{\mathrm{f}}-\varepsilon_{\mathrm{d}}\right) x}{\lambda}\right)$.

The result is plotted in figure 3 . When $\lambda$ is large (fast case) :

$$
n_{\mathrm{d}}(\infty) \simeq \frac{1}{2}+\frac{\varepsilon_{\mathrm{f}}-\varepsilon_{\mathrm{d}}}{\lambda}
$$

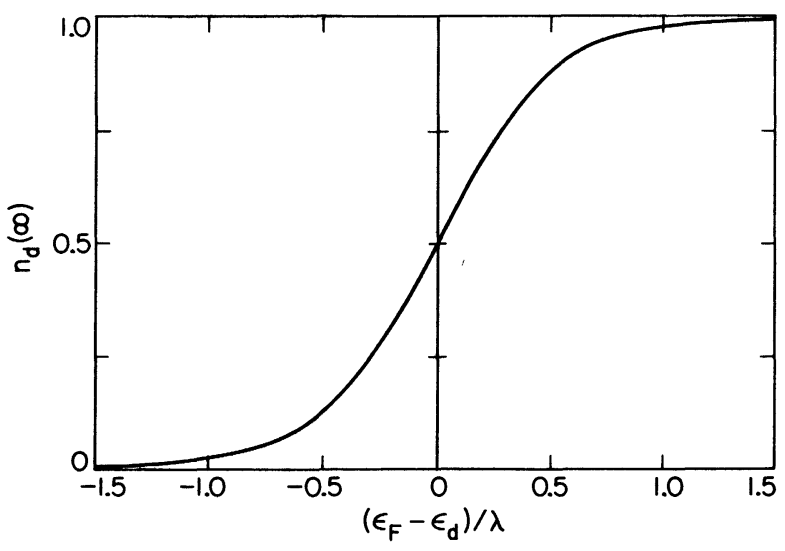

Fig. 3. - The d-level occupation $n_{\mathrm{d}}(\infty)$ in the ionization problem with $\Delta(t) \propto \mathrm{e}^{-\lambda t}$ (see eq. (59)).

When $\lambda$ is small (slow case) :

$$
\begin{array}{r}
n_{\mathrm{d}}(\infty) \simeq\left\{\begin{array}{l}
0 \\
1
\end{array} \pm \frac{2}{\pi} \exp \left(-\frac{\pi\left|\varepsilon_{\mathrm{f}}-\varepsilon_{\mathrm{d}}\right|}{\lambda}\right)\right. \\
\text { for }\left\{\begin{array}{l}
\varepsilon_{\mathrm{f}}<\varepsilon_{\mathrm{d}} \\
\varepsilon_{\mathrm{f}}>\varepsilon_{\mathrm{d}}
\end{array}\right.
\end{array}
$$

In general, when an atom leaves a surface, $V_{\mathrm{d} k}(x)$ behaves approximately as $V_{\mathrm{dk}}(0) \exp (-x / a)$, where $a$ is a characteristic length of the order of the width of the surface (a few Angstroms). For a constant speed of the atom, we then have an exponentially decreasing $V_{\mathrm{d} k}(t)$, and the non-adiabatic description must be used.

For a small departure from adiabaticity, eq. (61) appears very similar to the results obtained in reference [1]. Details will be given in a forthcoming publication.

5. Friction coefficients. - When an atom is moving in a metal or near the surface of a metal, it experiences friction forces. We shall discuss here only the friction forces which are due to the electrons. Their expression is usually obtained from a general formula involving the correlation function of the generalized force of the system. We present here an alternative derivation. We will then discuss some applications.
5.1 Calculation of THE FRICTION FORCE. - We assume that the system is described by the Hamiltonian (26) where $V(t)$ is slowly varied. We are interested in the behaviour of the system at some instant $t=0$ (the choice of this instant being only a matter of convenience) and treat $\delta V(t)=V(t)-V(0)$ as the perturbation. For times not too far from 0 , the system is not far from the instantaneous equilibrium and the perturbed Green's function $\tilde{G}$ departs little from the unperturbed one $G$ corresponding to the time-independent Hamiltonian $\mathscr{H}=\mathscr{H}(0)\left({ }^{4}\right)$. Since $\widetilde{G}^{\mathrm{a}}$ obeys a Dyson equation, we have

$$
\begin{aligned}
\widetilde{G}^{\mathrm{a}} & =G^{\mathrm{a}}+G^{\mathrm{a}} \delta V \tilde{G^{\mathrm{a}}} \\
& \simeq G^{\mathrm{a}}+G^{\mathrm{a}} \delta V G^{\mathrm{a}}
\end{aligned}
$$

retaining only the first order in $\delta V$. Of course a similar relation holds for $\widetilde{G}^{\mathrm{r}}$. Using the expression of $\tilde{G}$ in terms of $\tilde{G}^{\mathrm{a}}$ and $\tilde{G}^{\mathrm{r}}$, we obtain, to first order in $\delta V$ :

$$
\widetilde{G}=G+G^{\mathrm{a}} \delta V G+G \delta V G^{\mathrm{r}}-G^{\mathrm{a}} \delta V G^{\mathrm{r}} .
$$

In order to calculate the friction force, we write the time derivative of the energy :

$$
\begin{aligned}
\dot{E}(0) & =\sum_{k k^{\prime}} \dot{V}_{k k^{\prime}}(0)\left\langle c_{k}^{+}(0) c_{k^{\prime}}(0)\right\rangle \\
& =-i \sum_{k k^{\prime}} \dot{V}_{k k^{\prime}}(0) \widetilde{G}_{k^{\prime} k}\left(0,0^{+}\right) \\
& =\operatorname{Im} \operatorname{Tr}\left[\dot{V}(0) \tilde{G}\left(0,0^{+}\right)\right] .
\end{aligned}
$$

To lowest order, $\tilde{G}=G$ and we get the adiabatic limit :

$$
\begin{aligned}
\dot{E}_{\mathrm{ad}} & =\operatorname{Im} \operatorname{Tr} \dot{V} G\left(0^{-}\right) \\
& =-\frac{1}{\pi} \operatorname{Im} \operatorname{Tr} \int_{-\infty}^{\varepsilon_{\mathrm{f}}} \mathrm{d} \omega \dot{V} G^{\mathrm{a}}(\omega)
\end{aligned}
$$

It is instructive for the discussion later to transform this expression somewhat. Formally,

$$
-\frac{1}{\pi} \operatorname{Im} \dot{V} G^{\mathrm{a}}(\omega)=\dot{V} \delta(\omega-\mathscr{H}) .
$$

Under the trace, we can write :

$$
\dot{H} \delta(\omega-\mathscr{H})=-\frac{\mathrm{d}}{\mathrm{d} t} \theta(\omega-\mathscr{H})
$$

though $\mathcal{H}$ and $\dot{\mathcal{J}}$ do not commute. Defining :

$$
\eta(\omega)=\pi \operatorname{Tr} \theta(\omega-\mathscr{H}) \bmod \pi
$$

which is, according to Friedel's rule, the sum of the phase shifts in the case of a scattering problem, we may express $\dot{E}_{\text {ad }}$ as :

$$
\dot{E}_{\mathrm{ad}}=-\frac{1}{\pi} \int_{-\infty}^{\varepsilon_{\mathrm{f}}} \mathrm{d} \omega \dot{\eta}(\omega) .
$$

$\left({ }^{4}\right)$ For convenience we use a slightly different notation here from that used in the preceding sections. 
With this energy variation there is associated a conservative force $\mathbf{F}_{\mathrm{ad}}$ which is applied to the moving atom :

$$
\mathbf{F}_{\mathrm{ad}}=\frac{1}{\pi} \int_{-\infty}^{\varepsilon_{\mathrm{f}}} \mathrm{d} \omega \nabla \eta(\omega)
$$

The first correction to $\dot{E}_{\text {ad }}$ is obtained by substituting for $\widetilde{G}$ in (65) the last three terms of (64). The third one vanishes when we take the imaginary part, and we get :

$$
\dot{E}_{1}(0)=\operatorname{Im} \operatorname{Tr} \dot{V}(0)\left[G^{\mathrm{a}} \delta V G+G \delta V G^{\mathrm{r}}\right](0,0) .
$$

Assuming a linear variation of $\delta V$ with time; i.e., $\delta V(t)=V(0) t$, we find the friction term :

$$
\begin{aligned}
\dot{E}_{\mathrm{f}}(0)= & \operatorname{Im} \operatorname{Tr} \int \mathrm{d} \tau \tau \dot{V}(0) G(\tau) \dot{V}(0) \times \\
\times & {\left[G^{\mathrm{a}}(-\tau)-G^{\mathrm{r}}(-\tau)\right] } \\
= & \operatorname{Re} \operatorname{Tr} \int \frac{\mathrm{d} \omega}{2 \pi} \dot{V}(0) G(\omega) \dot{V}(0) \times \\
\times & \frac{\mathrm{d}}{\mathrm{d} \omega}\left[G^{\mathrm{a}}(\omega)-G^{\mathrm{r}}(\omega)\right] \\
=\operatorname{Tr} \int \frac{\mathrm{d} \omega}{4 \pi} \dot{V}(0)[G(\omega) & \left.-G^{+}(\omega)\right] \dot{V}(0) \\
& \times \frac{\mathrm{d}}{\mathrm{d} \omega}\left[G^{\mathrm{a}}(\omega)-G^{\mathrm{r}}(\omega)\right]
\end{aligned}
$$

where $G_{k k^{\prime}}^{+}(\omega)=G_{k^{\prime} k}^{*}(\omega)$. Remember that $G^{\mathrm{r}}=G^{\mathrm{a}+}$. Finally we use the fact that $G(\omega)=G^{\mathrm{a}}(\omega)$ for $\omega>\varepsilon_{\mathrm{f}},=G^{\mathrm{r}}(\omega)$ for $\omega<\varepsilon_{\mathrm{f}}$. An elementary integration yields :

$$
\dot{E}_{\mathrm{f}}=-\frac{1}{4 \pi} \operatorname{Tr}\left[\dot{V}\left(G^{\mathrm{a}}\left(\varepsilon_{\mathrm{f}}\right)-G^{\mathrm{r}}\left(\varepsilon_{\mathrm{f}}\right)\right)\right]^{2} .
$$

Formally, this can be written

$$
\dot{E}_{\mathrm{f}}=\pi \operatorname{Tr}\left[\dot{V} \delta\left(\varepsilon_{\mathrm{f}}-\dddot{H}\right)\right]^{2} .
$$

Let $|\varepsilon v\rangle$ be the eigenvectors of $\mathscr{H}$, where $\varepsilon$ is the eigenvalue and $v$ discriminates between degenerate states. The normalization is taken as

$$
\left\langle\varepsilon v \mid \varepsilon v^{\prime}\right\rangle=\delta\left(\varepsilon-\varepsilon^{\prime}\right) \delta_{v v^{\prime}} .
$$

Then $\dot{E}_{\mathrm{f}}$ becomes :

$$
\dot{E}_{\mathrm{f}}=\pi \sum_{v, v^{\prime}}\left|\left\langle\varepsilon_{\mathrm{f}} v|\dot{V}| \varepsilon_{\mathrm{f}} v^{\prime}\right\rangle\right|^{2} .
$$

This corresponds to a friction force :

$$
\mathbf{F}_{\mathrm{f}}=-\pi \sum_{v, v^{\prime}}\left|\left\langle\varepsilon_{\mathrm{f}} v|\nabla V| \varepsilon_{\mathrm{f}} v^{\prime}\right\rangle\right|^{2} \mathbf{u}
$$

where $\mathbf{u}$ is the velocity of the particle.

Thus we recover the result usually derived from the general formula relating the friction coefficient to the force-force correlation function; e.g., compare (74) with eq. (12) of reference [4].

5.2 Applications. - We can distinguish two contributions to $\dot{E}_{\mathrm{f}}$ : the contribution $\dot{E}_{\mathrm{f}}^{\mathrm{D}}$ from diagonal terms and the contribution $\dot{E}_{\mathrm{f}}^{\mathrm{ND}}$ from the offdiagonal terms. Of course $\dot{E}_{\mathrm{f}}^{\mathrm{ND}}$ could be eliminated if we were able to find a basis in which $\dot{V}$ is diagonal. For instance, if $V$ and $V$ are both centrally symmetric, such a basis is that of the spherical harmonics $v=\{l, m\}$.

Let us first consider $\dot{E}_{\mathrm{f}}^{\mathrm{D}}$ :

$$
\dot{E}_{\mathrm{f}}^{\mathrm{D}}=\pi \sum_{v}\left|\left\langle\varepsilon_{\mathrm{f}} v|\dot{V}| \varepsilon_{\mathrm{f}} v\right\rangle\right|^{2}
$$

and define, similarly to (67) :

$$
\eta_{v}^{\mathrm{f}}=\pi \underset{(v)}{\operatorname{Tr}} \theta\left(\varepsilon_{\mathrm{f}}-\mathscr{H}\right) \bmod \pi
$$

where the trace is over $v$-states. For a centrally symmetric scattering problem, $v=\{l, m\}$ and $v_{l}^{f}$ is the $l$-phase shift at the Fermi level. For the same reasons as in the calculation of $\dot{E}_{\text {ad }}$ above, we can write :

$$
\dot{E}_{\mathrm{f}}^{\mathrm{D}}=\frac{1}{\pi} \sum_{v}\left(\dot{\eta}_{v}^{\mathrm{f}}\right)^{2} .
$$

In particular, for a centrally symmetric potential :

$$
\dot{E}_{\mathrm{f}}^{\mathrm{D}}=\frac{1}{\pi} \sum_{l}(2 l+1)\left(\dot{\eta}_{l}^{\mathrm{f}}\right)^{2} .
$$

An example where, on the contrary, $\dot{E}_{\mathrm{f}}$ appears naturally through $\dot{E}_{\mathrm{f}}^{\mathrm{ND}}$ is that of an atom moving through an infinite electron gas with a velocity $\mathbf{u}$. Then

$$
\dot{V}=-\mathbf{u} \cdot \operatorname{grad} V=-u \cos \theta \frac{\mathrm{d} V}{\mathrm{~d} r}
$$

$V$ has matrix elements between the spherical harmonics $\{l, m\}$ and $\{l \pm 1, m\}$. In terms of the phase shifts, we obtain, $m_{\mathrm{e}}$ being the electron mass :

$$
\dot{E}_{\mathrm{f}}=\frac{4 m_{\mathrm{e}} u^{2} \varepsilon_{\mathrm{f}}}{3 \pi} \sum_{l}(l+1) \sin ^{2}\left(\eta_{l+1}^{\mathrm{f}}-\eta_{l}^{\mathrm{f}}\right) .
$$

This result is obviously linked to the formula for the resistivity due to the same atom in the electron gas [5].

For the Anderson Hamiltonian which was discussed in section 4 , the friction coefficient is thus proportional to $\sin ^{2} \eta^{\mathrm{f}}$ when the atom moves in the bulk. This result can be extended, at least qualitatively, to the case of an atom moving along the surface. When, on the contrary, the atom leaves the surface, the main effect comes from the variation of $V_{\mathrm{d} k}$ in magnitude. In the approximation $V_{\mathrm{d} k}(t)=V_{\mathrm{d} k} u(t)$, which implies that $V$ is taken to remain centrally symmetric, the friction coefficient is proportional to $\left(\mathrm{d} \eta^{\mathrm{f}} / \mathrm{d} x\right)^{2}, x$ being the distance to the surface. 
Thus we have essentially two regimes for the friction force, depending on the kind of variation of the potential involved.

6. Conclusion. - We have presented a framework for the discussion of localized time-dependent onebody perturbations in metals. Let us summarize the main results :

1) The Green's functions $G_{k k^{\prime}}\left(t, t^{\prime}\right)$ can be expressed in terms of the advanced and retarded functions (eq. (36)). This reduces the many-electron problem to the solution of a one-body problem.

2) The Green's functions can be used to calculate the occupancies of given states. This permits the calculation of the ionization probability of atoms leaving surfaces, and charge exchange between metals and atoms or ions.

3) We have discussed the friction forces which are experienced by moving atoms and the two extreme regimes which can occur. Applications to surfaces are obvious $\left({ }^{5}\right)$. The electronic stopping power of channeled particles can be interpreted within the same framework.

Acknowledgments. - The authors acknowledge a useful discussion with Professor W. Kohn.

$\left({ }^{5}\right)$ This concerns only the friction due to electrons. Friction due to the vibrations of the solid have to be added. The electronic contribution (79) or (80) can be of the same order of magnitude as the phonon contribution.

\section{References}

[1] Cobas, A. and Lamb, W. E., Phys. Rev. 65 (1944) 327.

[2] Keldysh, L. V., Sov. Phys. JETP 20 (1965) 1018.

[3] Nozières, Ph. and DE Dominicis, C. T., Phys. Rev. 178 (1969) 1097.

[4] Blandin, A., Hone, D. and Nourtier, A., J. Physique Lett. 36 (1975) L-109.

[5] Toulouse, G., Proceedings of the International School of Physics Enrico Fermi Course LVIII 759 (1974).
[6] See, for example, the review paper : Zwanzig, R., Annu. Rev. Phys. Chem. 16 (1965) 67.

[7] See, for example, D'Agliano, E. G., Schaich, W. L., Kumar, P. and SUHL, H., in Collective Properties of Physical Systems, Nobel Symposium 24 (1973) 200. 